\title{
Tomographic reconstruction of the single-photon Fock state by high-frequency homodyne detection
}

\author{
Alessandro Zavatta, Silvia Viciani, and Marco Bellin * \\ Istituto Nazionale di Ottica Applicata (INOA), \\ L.go E. Fermi, 6, I-50125, Florence, Italy
}

(Dated: July 3, 2018)

\begin{abstract}
A single-photon Fock state has been generated by means of conditional preparation from a twophoton state emitted in the process of spontaneous parametric down-conversion. A recently developed high-frequency homodyne tomography technique has been used to completely characterize the Fock state by means of a pulse-to-pulse analysis of the detectors' difference photocurrent. The density matrix elements of the generated state have been retrieved with a final detection efficiency of about 57\%. A comparison has been performed between the phase-averaged tomographic reconstructions of the Wigner function as obtained from the measured density-matrix elements and from a direct Abel transform of the homodyne data. The ability of our system to work at the full repetition rate of the pulsed laser $(82 \mathrm{MHz})$ substantially simplifies the detection scheme, allowing for more "exotic" quantum states to be generated and analyzed.
\end{abstract}

PACS numbers: 03.65.Wj, 03.65.Ud, 42.50.Dv

\section{INTRODUCTION}

Homodyne detection is a widely used method to obtain tomographic reconstructions of classical and quantum optical fields in the $\mathrm{cw}$ and pulsed regimes. It consists in mixing the unknown signal field with a strong local oscillator (LO) field on a beam splitter whose outputs are then detected by proportional photodetectors [1]. The difference in the photocurrents produced by the two detectors is proportional to the electric field quadrature selected by varying the relative phase between the LO and the signal field. From a collection of field quadratures it is then possible to reconstruct the quantum state by means of tomographic techniques. Vacuum, thermal, coherent and squeezed states have been successfully analyzed, in the frequency-domain, by means of a spectral analysis of the difference photocurrent [2, 3], and in the time domain, with the measurement of integrated photocurrents on isolated pulses, in order to achieve a full reconstruction of the unknown field states [4, 5, 6].

Recently, a pulsed homodyne technique 7] has been successfully applied to the first complete quantum state reconstruction of single-photon Fock states of the electromagnetic field [8], i.e. states containing exactly one quantum of field excitation. Such states are an important workbench for the test and the analysis of nonclassical behavior and represent a fundamental building block for the implementation of efficient linear quantum computation. Time-domain homodyne detection schemes as those used in [7, 8] are however quite demanding from the experimental point of view, since they require very low electronic noise and high subtraction efficiencies over very large frequency bandwidths (from dc to a few times the repetition rate of the laser). Mostly due to these technical limitations, all the existing applications of the latter technique have been limited to pulse repetition rates well below the megahertz. The measurements reported in [7, 8] indeed made use of a pulse picker to lower the laser repetition frequency, and the low state production rates implied very long acquisition times in order to store enough data for a single accurate tomographic reconstruction. More recent works by the same group [9, 10] have adopted a different technique, where the local oscillator pulses are sent "on demand" to the homodyne detector only when needed; this has contributed to significantly increase their acquisition rates, although at the price of a more complicated setup and lower efficiencies.

Our group has recently developed an homodyne setup which is able to perform high-frequency (with repetition rates up to about $100 \mathrm{MHz}$ ) time-domain analysis of quantum states of light 11]. Its capabilities were first demonstrated by performing a tomographic reconstruction of the Wigner function of weak coherent states and by showing the possibility of a gated-mode acquisition for conditional measurements. Here we present the first complete and efficient tomographic reconstruction of a single-photon Fock state based on such a timedomain, pulsed optical homodyne apparatus operating at the high repetition frequencies characteristic of commonly used mode-locked laser systems. By allowing us to perform ultrafast pulse-selective measurements, this apparatus exhibits two fundamental advantages over alternative schemes working at lower repetition rates: on one side, thanks to the much shorter acquisition times, the stability requirements of the overall experimental system are loosened; on the other, if long-term stability of the setup is available, states exhibiting stronger nonclassical features (usually characterized by smaller generation probabilities) can be efficiently analyzed. 


\section{GENERATION OF SINGLE-PHOTON FOCK STATES}

A single-photon Fock state can be generated by means of a conditional state preparation on the two-photon wavefunction emitted in the process of spontaneous parametric down-conversion (SPDC) (see Refs. 12, 13] and references therein). Such a state is written as

$$
\begin{aligned}
|\Psi\rangle= & |0\rangle_{s}|0\rangle_{t}-\int \mathrm{d}^{3} k_{s} \mathrm{~d} \omega_{s} \mathrm{~d}^{3} k_{t} \mathrm{~d} \omega_{t} \psi\left(\mathbf{k}_{s}, \omega_{s}, \mathbf{k}_{t}, \omega_{t}\right) \\
& \times\left|\mathbf{k}_{s}, \omega_{s}\right\rangle_{s}\left|\mathbf{k}_{t}, \omega_{t}\right\rangle_{t}
\end{aligned}
$$

where the function $\psi\left(\mathbf{k}_{s}, \omega_{s}, \mathbf{k}_{t}, \omega_{t}\right)$ describes the spatial and spectral properties of the two-photon state (biphoton) and the subscripts $s$ and $t$ correspond to the signal and trigger (idler) photons.

In order to non-locally select a pure state on the signal channel, trigger photons must undergo narrow spatial and frequency filtering before being detected by a single photon counter [12,14]. The conditional non-local preparation of the signal state is thus described by the density operator $\hat{\rho}_{s}$ given by the partial trace of the product between the SPDC-state density operator and the measurement density operator $\hat{\rho}_{t}$ :

$$
\hat{\rho}_{s}=\operatorname{Tr}\left\{\hat{\rho}_{t}|\Psi\rangle\langle\Psi|\right\}_{t}
$$

where

$$
\hat{\rho}_{t}=\int \mathrm{d}^{3} k_{t} \mathrm{~d} \omega_{t} T\left(\mathbf{k}_{t}, \omega_{t}\right)\left|\mathbf{k}_{t}, \omega_{t}\right\rangle_{t}\left\langle\mathbf{k}_{t},\left.\omega_{t}\right|_{t}\right.
$$

and $T(\mathbf{k}, \omega)$ is the transmission function of the spatial and spectral filter placed along the trigger photon path. Note that the nonlocally-prepared signal state will only approach a pure state if the filter transmission function $T(\mathbf{k}, \omega)$ is much narrower than the momentum and spectral widths of the pump beam generating the SPDC pair. Accordingly, one can define a purity parameter as $P=\operatorname{Tr}\left(\hat{\rho}_{s}^{2}\right)$ which, in the spectral domain, is expressed as

$$
P_{t}=\frac{1}{\sqrt{1+\sigma_{f}^{2} / \sigma_{p}^{2}}}
$$

where Gaussian profiles with widths $\sigma_{p}$ and $\sigma_{f}$ are assumed for the pump power spectrum and for the trigger filter transmission, respectively. It is easy to see how the spectral purity parameter $P_{t}$ approaches unity when $\sigma_{f} \ll \sigma_{p}$. In such a condition the strong spectral filtering performs a non-local selection of a pure signal state whose properties are defined by the pump as shown in [13, 15]. Similarly, the spatial purity parameter is found to be [12]:

$$
P_{s}=\frac{1}{1+\kappa_{i}^{2} / \kappa_{p}^{2}}
$$

where $\kappa_{i}$ and $\kappa_{p}$ are the beam widths in the momentum space for the idler spatial filter and for the pump, respectively.

\section{EXPERIMENTAL SETUP}

A mode-locked Ti:sapphire laser, emitting 1-2 ps long pulses at $786 \mathrm{~nm}$ with a repetition rate of $82 \mathrm{MHz}$ is used as the primary source for the experiment, schematically drawn in Fig 11 The laser pulses are first frequency doubled in a 13-mm long LBO crystal which produces the pump pulses centered at $393 \mathrm{~nm}$, with a mean power of about $100 \mathrm{~mW}$. The UV pump pulses, after a spatial mode cleaner, are then slightly focused, with a beam waist $w_{p}=220 \mu \mathrm{m}$, and the waist position is carefully located inside a 3-mm thick, Type I BBO crystal cut for collinear degenerate SPDC. The crystal is adjusted in order to obtain a signal-trigger cone beam with an angle of $\sim 3^{\circ}$. Signal and trigger photon pairs are then selected by means of irises placed at $70 \mathrm{~cm}$ from the output face of the crystal.

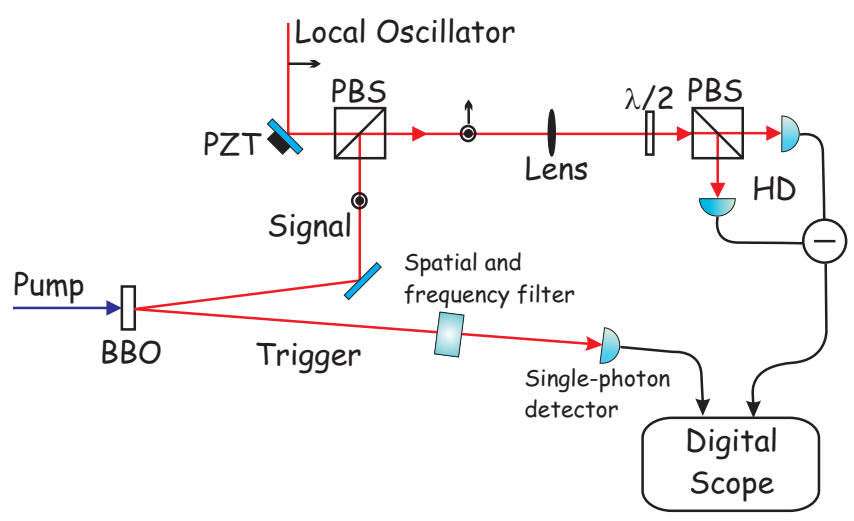

FIG. 1: Experimental apparatus: BBO downconverter crystal, PZT piezoelectric transducer, PBS polarizing beamsplitter, $\lambda / 2$ half-wave plate, HD homodyne detector.

The trigger beam is directed to the state preparation channel, where it is spectrally filtered by means of a pair of etalon interference filters and is then coupled into a single-mode fiber connected to a single photon counting module with an active-area diameter of $200 \mu \mathrm{m}$ (PerkinElmer SPCM AQR-14). The typical trigger count rate after spectral and spatial filtering is of about $500 \mathrm{~s}^{-1}$, with a dark count rate of the order of $10 \mathrm{~s}^{-1}$ when both are gated with the laser pulses.

The combination of the two etalon cavities yields a final spectral width for the trigger channel of about 50 $\mathrm{GHz}$. By comparing this with a measured pump width of $430 \mathrm{GHz}$, we estimate a spectral purity parameter of $P_{t}=0.98$. However, due to the second harmonic generation process, the LO spectral width remains $\sqrt{2}$ times narrower than that of the selected single-photon state. 
The degree of mode-matching between the LO and the single-photon state cannot be improved by filtering the LO beam and might be increased only by inserting a spectral filter on the UV pump. However, this solution would also drastically lower the count rate on the trigger detector and has not been implemented yet.

Filtering in the spatial domain is achieved by means of the single-mode fiber and our experimental parameters lead to a spatial purity parameter $P_{s}=0.86$, which again means that the conditionally prepared single-photon state mode is essentially determined by the pump characteristics. The LO beam is spatially mode-matched to the fiber-selected signal photon mode by the insertion of appropriate lens combinations along its path. In order to finely adjust the alignment and the synchronization between the signal and LO pulses, we use the stimulated beam produced by injecting a seed pulse into the crystal. Under appropriate conditions [12], the amplified seeded beam can spatially well simulate the selected signal beam and can be used for alignment purposes.

The LO pulses are obtained by splitting a small portion of the laser emission, and their polarization is rotated by a half-wave plate in order to overlap them with the conditionally-prepared single-photon signal beam onto a polarizing beam splitter (PBS). One of the steering mirrors is mounted on a piezoelectric transducer in order to vary and control the LO phase. A 300-mm focal-length lens, an additional $\lambda / 2$ wave plate, and a second polarizing beam splitter are then used to accurately mix the two field modes and focus them onto the two photodiodes of the homodyne detector.

\section{HIGH-FREQUENCY BALANCED HOMODYNE DETECTION}

Two photodiodes (Hamamatsu S3883, with active area $1.7 \mathrm{~mm}^{2}$ ) are connected to the positive and negative inputs of an operational amplifier providing a gain of about 10. The bias voltage of the two photodiodes is optimized in order to provide the maximum extinction in the difference signal when the optical power impinging on the two is well balanced. The amplifier output is AC-coupled (cut-off frequency of $1 \mathrm{MHz}$ at $-3 \mathrm{~dB}$ ) to a second amplifier with gain $\mathrm{G}=100$ and bandwidth extending from dc to $300 \mathrm{MHz}$, before being sent to a digital scope with an analog bandwidth of $1 \mathrm{GHz}$, a sampling rate of 10 Gsamples/s and a vertical resolution of 8 bits.

In order to decrease the effect of the dark counts in the single-photon trigger detector, a strict coincidence with the signal coming from the laser pulse train is used as the trigger for the acquisition of the homodyne signal. Although this slightly reduces the trigger count rate, it is effective in increasing the ratio of "true" to "false" trigger events to more than $99 \%$.

A single temporal frame composed of 250 acquisition points is stored in the oscilloscope memory whenever a trigger event occurs. Each of these frames contains two consecutive LO pulses where only the first one is coincident with the detection of a trigger photon and contains the "single-photon information", while the second one can be used for the measurement of the reference vacuum state. About 5000 frames can be stored in a sequence at a maximum rate of 160,000 frames per second. With an average rate of trigger counts of about 300 $\mathrm{s}^{-1}$, this means that each sequence is acquired in about 15-20 s. Each sequence of frames is then transferred to a personal computer where the areas of the pulses are measured and their statistic distributions are analyzed in real time. The acquisition of two nearby LO pulses in each frame allows the simultaneous analysis of the "signal" pulses, namely those that have interfered with the signal photons, and of the "dark" pulses, so that both the Fock-state and the LO shot-noise distributions are immediately available from a single sequence, independent from low-frequency fluctuations in the system. The acquisition and analysis of a sequence of 5000 signal and dark pulses yields an experimental Wigner marginal distribution in just about $30 \mathrm{~s}$.

It is interesting to compare these acquisition times with those reported in [8] by the Constance group for the first tomographic reconstruction of the single-photon Fock state. Due to the limited bandwidth of their homodyne detector $(1 \mathrm{MHz})$, the overall pulse rate had to be lowered to about $800 \mathrm{kHz}$ by means of a pulse picker at the exit of the laser. In that case about 12,000 experimental data points were acquired in a 14-hour experimental run in order to obtain an accurate phase-averaged marginal distribution of the quantum state. Our setup now allows the same kind of acquisition to be performed in just about one minute, with a gain of almost three orders of magnitude in the measurement time. It is clear that such a speed-up can help to loosen some of the constraints on the overall stability of the generation system and, in particular, on the interferometric stability of the beam paths which is needed when phase-dependent quantum states are to be analyzed. On the other hand, by greatly increasing the overall number of available LO pulses in a reasonable experimental time interval, our scheme will allow us to investigate much rarer and more exotic events, such as those involving higher photon numbers 11, 16].

Note that recent experiments from the same group 9, 10] have seen the adoption of a clever solution to the limited acquisition rate caused by the low detector bandwidth. Instead of reducing the whole experimental repetition rate, the pulse picker has been placed only in the LO path and activated "on demand" upon a trigger detection event. This has allowed to keep reasonable pair production rates and to obtain a final acquisition rate similar to the one presented here. However, the introduction of the pulse picker, besides significantly complicating the experimental setup, has the important 
drawback that the trigger delay of the shutter in the LO path has to be compensated by a long (about $15 \mathrm{~m}$ ) optical delay line on the signal photon path in order for the two to reach the mixing beam-splitter at the same time. This clearly introduces additional losses and may degrade both the mode quality and the accurate phase stability of the fragile quantum state.

The performances of our detector were verified with a preliminary measurement of the laser shot-noise by analyzing the variances of the "dark" pulse areas as a function of the LO power. A linear behavior is preserved over a wide range of LO powers, extending up to $9 \mathrm{~mW}$, and with a signal to electronic-noise ratio of about $12 \mathrm{~dB}$ when the device is operated at the optimum LO power of $7 \mathrm{~mW}$.

\section{QUANTUM STATE RECONSTRUCTION}

Balanced homodyne detection allows the measurement of the signal electric field quadratures $x_{\theta}$ as a function of the relative phase $\theta$ imposed between the $\mathrm{LO}$ and the signal. By performing a series of homodyne measurements on equally-prepared states it is possible to obtain the probability distributions $p(x, \theta)$ of the quadrature operator $\hat{x}_{\theta}$ that correspond to the marginals of the Wigner quasi-probability distribution $W(x, y)$ [17]:

$$
p(x, \theta)=\int_{-\infty}^{+\infty} W(x \cos \theta-y \sin \theta, x \sin \theta+y \cos \theta) \mathrm{d} y .
$$

In principle, given a sufficient number of quadrature distributions for different values of $\theta$, one is able to reconstruct the Wigner function by inverting Eq. (6). In the case of Fock states, some simplification in the reconstruction procedures can be accomplished thanks to the phase-invariant nature of the Wigner function [8]. Only one phase-averaged marginal distribution is required for the reconstruction in this case and, from the experimental point of view, this also means that the stabilization of the LO phase is not needed. Furthermore, the Abel transform, relying on the cylindrical symmetry of the problem, can be used instead of the Radon transform in order to reconstruct a section of the Wigner function $W(r)$ [18], as given by

$$
W(r)=-\frac{1}{\pi} \int_{r}^{+\infty} \frac{\partial p_{\text {av }}(x)}{\partial x} \frac{\mathrm{d} x}{\sqrt{x^{2}-r^{2}}}
$$

where $p_{\text {av }}(x)$ is the phase-averaged marginal distribution. The numerical implementation of this method is however extremely sensitive to noise and a large number of data is required to obtain a reliable result. Since this reconstruction procedure involves the application of numerically-unreliable methods, the group of D'Ariano has developed an alternative precise technique that per-

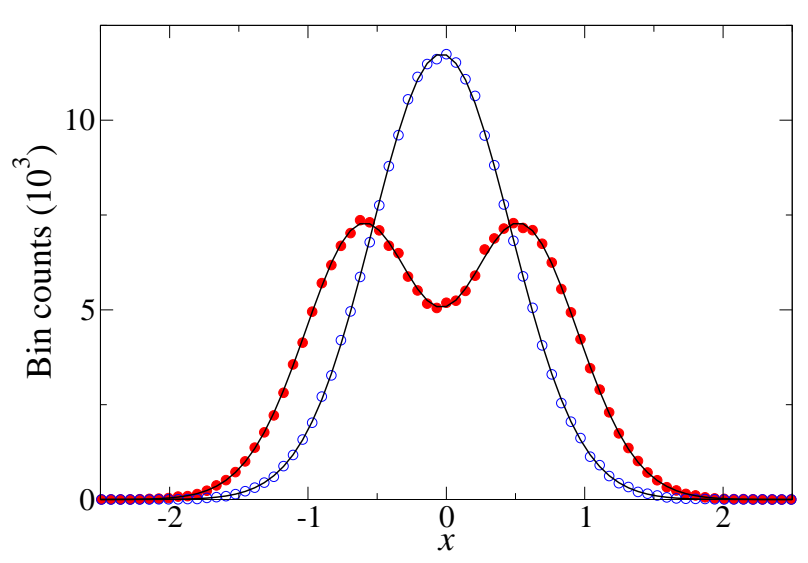

FIG. 2: Vacuum (open circles) and signal (filled circles) phaseaveraged quadrature field distributions. The solid lines are fitted curves. The quadrature is normalized to the vacuum.

mits the reconstruction of the density matrix elements directly from the homodyne data 19].

The distributions of the acquired pulse areas for the vacuum and the single-photon state are shown in Fig. 2 where each histogram is obtained from the analysis of about 200,000 acquired pulses. Such distributions represent the marginal integrals of the corresponding field state. The non-unity detection efficiency of the apparatus prevents us from observing the real single-photon Wigner function, and what we get instead is its convolution with the vacuum one. The convolution result is the well known $s$-parametrized quasi-probability distribution with the $s$ parameter scaled by the detection efficiency $\eta[18,20]$. The expression for the corresponding marginal distribution is:

$$
p(x ; \eta)=\sqrt{\frac{2}{\pi}}\left[1-\eta\left(1-4 x^{2}\right)\right] e^{-2 x^{2}}
$$

From a fit of the experimental distributions to the corresponding theoretical curves we obtain an overall detection efficiency of $\eta=0.574 \pm 0.002$.

The expected overall efficiency $\eta$ is given by the contribution of several terms:

$$
\eta=\eta_{\mathrm{hd}} \eta_{\mathrm{dc}} \eta_{\mathrm{mm}}
$$

where $\eta_{\text {hd }}=0.90$ is the efficiency of the homodyne detector including the PBS losses, while $\eta_{\mathrm{dc}}=0.99$ is the efficiency connected to the dark counts of the trigger detector. The mode-matching efficiency is given by $\eta_{\mathrm{mm}}=\eta_{\exp } \sqrt{P_{s} P_{t}}$, where $\sqrt{P_{s} P_{t}}$ depends on the tightness of the spatial and spectral filtering on the trigger channel and constitutes the upper limit for the mode matching efficiency achievable between a pure state (LO) and the non-locally selected single photon state. The term $\eta_{\exp }$ is connected with the non-ideal experimental conditions and can be estimated from the visibility of the interference fringes formed between the LO and the 


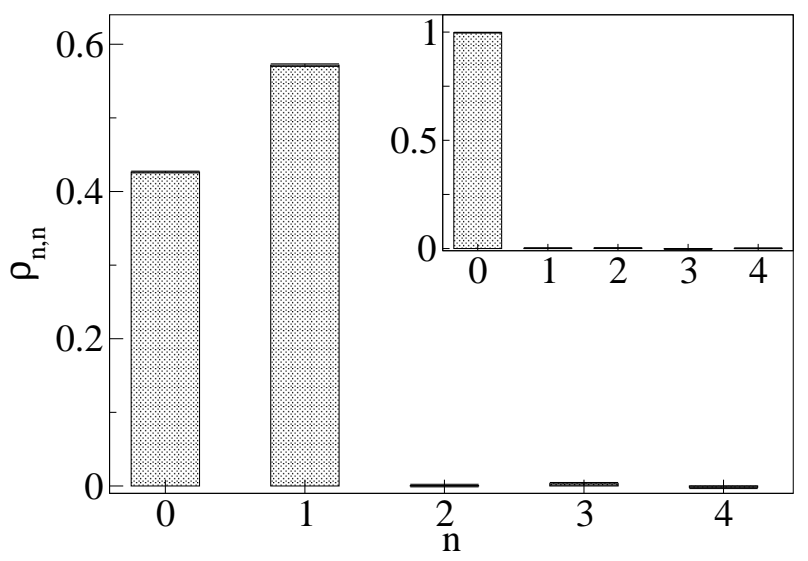

FIG. 3: Reconstructed diagonal elements of the density matrix for the single-photon state and the vacuum (inset).

stimulated radiation used for alignment 12]. We obtain $\eta_{\exp } \simeq 0.7$ and the overall efficiency is thus expected to be $\eta \simeq 0.6$, in good agreement with the value retrieved from the fit of the marginal data to the theoretical distributions.

In Fig. 3 the reconstructed diagonal elements $\rho_{n, n}$ of the density matrix are reported as obtained by averaging the so-called pattern functions over our data:

$$
\rho_{n, n}=\langle n|\hat{\rho}| n\rangle=\pi \int_{-\infty}^{+\infty} p_{\mathrm{av}}(x) f_{n n}(x) \mathrm{d} x .
$$

Here $f_{n n}(x)$ are the factorized pattern functions that can be numerically implemented following the numerical recipes given in Ref. 18, 21]. Figure 3 clearly shows the non-poissonian photon distribution of the reconstructed state, which is a statistical mixture of the single-photon Fock state with the vacuum state $\hat{\rho}=$ $\rho_{0,0}|0\rangle\left\langle 0\left|+\rho_{1,1}\right| 1\right\rangle\langle 1|$ with $\rho_{0,0}=0.426 \pm 0.001$ and $\rho_{1,1}=0.572 \pm 0.002$ in good agreement with the efficiency value found by fitting the marginal distributions.

As discussed by D'Ariano in [21, 22], the Wigner function can be obtained from the reconstructed densitymatrix elements in a dimensionally-truncated Hilbert space as:

$$
W(x, y)=\operatorname{Re} \sum_{d=0}^{M} e^{i d \arctan (y / x)} \sum_{n=0}^{M-d} \Lambda(n, d, x, y) \rho_{n, n+d}
$$

and

$$
\begin{array}{r}
\Lambda(n, d, x, y)=\frac{2(-1)^{n}}{\pi}\left[2-\delta_{d 0}\right]|2(x+i y)|^{d} \\
\times \sqrt{\frac{n !}{(n+d) !}} e^{-2\left(x^{2}+y^{2}\right)} L_{n}^{d}\left[4\left(x^{2}+y^{2}\right)\right]
\end{array}
$$

where $L_{n}^{d}(x)$ are Laguerre polynomials, $\delta$ is the Kronecker delta and $M$ is the maximal quantum number of

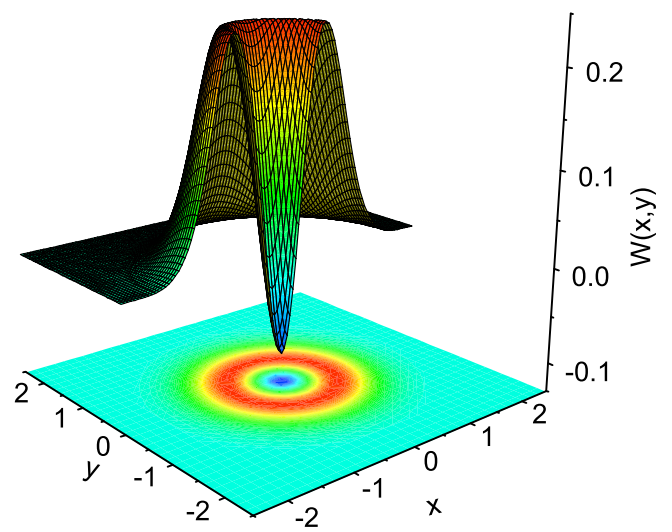

FIG. 4: Wigner function of the single-photon Fock state as obtained from the reconstructed density-matrix elements. The negativity of the distribution, a clear proof of the non-classical character of the state, is evident around the origin.

the reconstructed density matrix. In Fig 4 we show the Wigner function obtained from the first 10 reconstructed diagonal elements of the density matrix and by setting all the off-diagonal terms to zero thanks to the phaseinvariance of the state. The reconstructed Wigner function assumes negative values at the origin, showing the non-classical features of the single-photon state. We find $W_{\text {meas }}(0,0)=-0.0995$, which is quite close to the theoretical value of the Wigner function $W(0,0 ; \eta)=-0.0942$ obtained by using Eq. (11) with the mixed state defined by

$$
\hat{\rho}=(1-\eta)|0\rangle\langle 0|+\eta| 1\rangle\langle 1|
$$

with the efficiency value $(\eta=0.574)$ retrieved from the fit of the phase-averaged marginal distributions.

A comparison between the different methods of Wigner function reconstruction is presented in Fig. 5 where the $W(x, 0)$ section is plotted. Both the profile obtained by a direct implementation of the Abel transform on the acquired homodyne histograms using the basic algorithm of Nestor and Olsen 23] and a section of the Wigner function as reported in Fig 4 are shown, together with the curve retrieved using the mixed state of Eq. (13). It is evident that the direct reconstruction method based on the Abel transform is extremely sensitive to the noise present in the data and fails to accurately reproduce the expected vacuum-convoluted single-photon Wigner function. On the other hand, the reconstruction via the density-matrix elements is much smoother and extremely close to the expected profile.

Recently, alternative reconstruction techniques based on maximum-likelihood estimation methods have been proposed [24, 25] and used to analyze experimental 


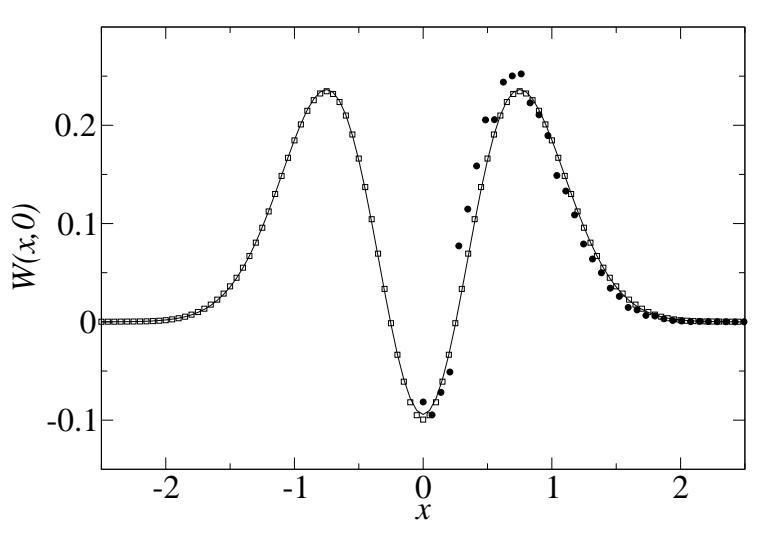

FIG. 5: $W(x, 0)$ section of the reconstructed Wigner function. Filled circles: Abel transform; empty squares: section of Fig 4 solid line: section corresponding to the mixed state of Eq. (13).

data 10]. Such methods can reconstruct the density matrix elements and the Wigner function with lower requirements on the number of data samples without a substantial increase in the statistical errors. The implementation of these methods might contribute to further decrease the acquisition time of our experiment whenever this becomes a limiting factor, i.e. when more exotic quantum states are analyzed.

\section{CONCLUSIONS}

We have applied a recently developed technique of time-domain, high-frequency homodyne detection to perform quantum tomographic reconstructions of the singlephoton Fock state. The scheme for the generation of the quantum field is based on remote state preparation from an entangled two-photon wavefunction emitted in the process of pulsed parametric down-conversion. By analyzing pulsed homodyne signals at the full repetition rate of the mode-locked source laser, this scheme allows us to perform full tomographic reconstructions in relatively short times. Furthermore, by allowing us to avoid the introduction of additional mechanisms of pulse selection, our scheme has the potential to achieve more compact realizations (which may be an issue when an accurate control of the relative phase in needed) and higher detection efficiencies in the analysis of quantum states. Finally, we have shown that the methods of Wigner function retrieval based on the direct analysis of the homodyne data are too sensitive to experimental noise and that much more accurate results can be obtained from the reconstructed density-matrix elements.

\section{ACKNOWLEDGMENTS}

This work has been partially supported by the Italian Ministry of University and Scientific Research (MIUR), under the FIRB contract RBNE01KZ94. The authors would also like to thank the Physics Department of the University of Florence for the kind hospitality and P. Poggi of the electronic workshop at INOA for the skillful implementation of the high-frequency detection electronics.

* Also at LENS and INFM, Florence, Italy; Electronic address: bellini@inoa.it

[1] S. Reynaud, A. Heidmann, E. Giacobono, and C. Fabre, in Progress in Optics, edited by E. Wolf (Elsevier, Amsterdam, 1992), vol. 30, p. 1.

[2] G. Breitenbach, S. Schiller, and J. Mlynek, Nature 387, 471 (1997).

[3] M. Vasilyev, S.-K. Choi, P. Kumar, and G. M. D'Ariano, Optics Lett. 23, 1393 (1998).

[4] D. T. Smithey, M. Beck, M. G. Raymer, and A. Faridani, Phys. Rev. Lett. 70, 1244 (1993).

[5] M. Crispino, G. D. Giuseppe, F. De Martini, and P. Mataloni, Fortschr. Phys. 48, 589 (2000).

[6] J. Wenger, R. Tualle-Brouri, and P. Grangier, Phys. Rev. Lett. 92, 153601 (2004).

[7] H. Hansen, T. Aichele, C. Hettich, P. Lodahl, A. I. Lvovsky, J. Mlynek, and S. Schiller, Opt. Lett. 26, 1714 (2001).

[8] A. I. Lvovsky, H. Hansen, T. Aichele, O. Benson, J. Mlynek, and S. Schiller, Phys. Rev. Lett. 87, 050402 (2001).

[9] A. I. Lvovsky and J. H. Shapiro, Phys. Rev. A 65, 033830 (2002).

[10] S. A. Babichev, B. Brezger, and A. I. Lvovsky, Phys. Rev. Lett. 92, 047903 (2004).

[11] A. Zavatta, M. Bellini, P. L. Ramazza, F. Marin, and F. T. Arecchi, J. Opt. Soc. Am. B 19, 1189 (2002).

[12] T. Aichele, A. I. Lvovsky, and S. Schiller, Eur. Phys. J. D 18, 237 (2002).

[13] S. Viciani, A. Zavatta, and M. Bellini, Phys. Rev. A 69, 053801 (2004).

[14] Z. Y. Ou, Quantum Semiclass. Opt. 9, 599 (1997).

[15] M. Bellini, F. Marin, S. Viciani, A. Zavatta, and F. T. Arecchi, Phys. Rev. Lett. 90, 043602 (2003).

[16] A. Montina and F. T. Arecchi, Phys. Rev. A 58, 3472 (1998).

[17] K. Vogel and H. Risken, Phys. Rev. A 40, 2847 (1989).

[18] U. Leonhardt, Measuring the quantum state of light (Cambridge University Press, Cambridge, England, 1997).

[19] G. M. D'Ariano, C. Macchiavello, and M. G. A. Paris, Phys. Rev. A 50, 4298 (1994).

[20] L. Mandel and E. Wolf, Optical Coherence and quantum optics (Cambridge University Press, Cambridge, England, 1995).

[21] G. M. D'Ariano, in Quantum Optics and Spectroscopy of Solids, edited by T. Hakioğlu and A. Shumovsky (Kluwer Academic Publishers, 1997), pp. 175-202. 
[22] G. M. D'Ariano, Quantum. Semiclass. Opt. 7, 693 (1995).

[23] C. J. Cremers and C. Birkebak, Appl. Opt. 5, 1057 (1966).

[24] K. Banaszek, G. M. D'Ariano, M. G. A. Paris, and M. F.
Sacchi, Phys. Rev. A 61, 010304 (1999).

[25] A. I. Lvovsky (2003), quant-ph/0311097. 